\title{
Localization and Register Sharing for Predicate Abstraction
}

\author{
Himanshu Jain^, Franjo Ivančić, Aarti Gupta, and Malay K. Ganai \\ NEC Laboratories America, 4 Independence Way, \\ Suite 200, Princeton, NJ 08540
}

\begin{abstract}
In the domain of software verification, predicate abstraction has emerged to be a powerful and popular technique for extracting finite-state models from often complex source code. In this paper, we report on the application of three techniques for improving the performance of the predicate abstraction refinement loop. The first technique allows faster computation of the abstraction. Instead of maintaining a global set of predicates, we find predicates relevant to various basic blocks of the program by weakest pre-condition propagation along spurious program traces. The second technique enables faster model checking of the abstraction by reducing the number of state variables in the abstraction. This is done by re-using Boolean variables to represent different predicates in the abstraction. However, some predicates are useful at many program locations and discovering them lazily in various parts of the program leads to a large number of abstraction refinement iterations. The third technique attempts to identify such predicates early in the abstraction refinement loop and handles them separately by introducing dedicated state variables for such predicates. We have incorporated these techniques into NEC's software verification tool F-SoFT, and present promising experimental results for various case studies using these techniques.
\end{abstract}

\section{Introduction}

In the domain of software verification, predicate abstraction [2, 7, 9, 11] has emerged to be a powerful and popular technique for extracting finite-state models from often complex source code. It abstracts data by keeping track of certain predicates on the data. Each predicate is represented by a Boolean variable in the abstract program, while the original data variables are eliminated. The application of predicate abstraction to large programs depends crucially on the choice and usage of the predicates. If all predicates are tracked globally in the program, the analysis often becomes intractable due to the large number of predicate relationships. In Microsoft's SLAM [4] toolkit, this problem is handled by generating coarse abstractions using techniques such as Cartesian approximation and the maximum cube length approximation [3]. These techniques limit the number of predicates in each theorem prover query. The refinement of the abstraction is carried out by adding new predicates. If no new predicates are found, the spurious behavior is due to inexact predicate relationships. Such spurious behavior is removed by a separate refinement algorithm called CONSTRAIN [1].

\footnotetext{
* The author is now at the School of Computer Science, Carnegie Mellon University.
} 
The BLAST toolkit [13] introduced the notion of lazy abstraction, where the abstraction refinement is completely demand-driven to remove spurious behaviors. Recent work [14] describes a new refinement scheme based on interpolation [8], which adds new predicates to some program locations only, which we will call henceforth localization of predicates. On average the number of predicates tracked at a program location is small and thus, the localization of predicates enables predicate abstraction to scale to larger software programs. In this paper we describe three novel contributions:

- Our first contribution is inspired by the lazy abstraction approach and the localization techniques implemented in BLAST. While BLAST makes use of interpolation, we use weakest pre-conditions to find predicates relevant at each program location. Given an infeasible trace $s_{1} ; \ldots ; s_{k}$, we find predicates whose values need to be tracked at each statement $s_{i}$ in order to eliminate the infeasible trace. For any program location we only need to track the relationship between the predicates relevant at that location. Furthermore, since we use predicates based on weakest pre-conditions along infeasible traces, most of the predicate relationships are obtained from the refinement process itself. This enables us to significantly reduce the number of calls to back-end decision procedures leading to a much faster abstraction computation.

- The performance of BDD-based model checkers depends crucially on the number of state variables. Due to predicate localization most predicates are useful only in certain parts of the program. The state variables corresponding to these predicates can be reused to represent different predicates in other parts of the abstraction, resulting in a reduction of the total number of state variables needed. We call this abstraction with register sharing. This constitutes our second technique which reduces the number of state variables, enabling more efficient model checking of the abstract models.

- While the above techniques speed up the individual computations and the model checking runs of the abstractions, they might result in too many abstraction refinement iterations. This can happen if the value of a certain predicate needs to be tracked at multiple program locations, i.e., if the predicate is useful globally or at least in some large part of the program. Since we add predicates lazily only along infeasible traces, the fact that a predicate is globally useful for checking a property will be learned only through multiple abstraction refinement iterations. We make use of a simple heuristic for deciding when the value of a certain predicate may need to be tracked globally or in a complete functional scope. If the value of a predicate needs to be tracked in a large scope, then it is assigned a dedicated state variable which is not reused for representing the value of other predicates in the same scope.

Further Related Work: Rusu et al. [20] present a framework for proving safety properties that combines predicate abstraction, refinement using weakest pre-conditions and theorem proving. However, no localization of predicates is done in their work. Namjoshi et al. [18] use weakest pre-conditions for extracting finite state abstractions, from possibly infinite state programs. They compute the weakest pre-conditions starting from an initial set of predicates derived from the specification, control guards etc. This process is iterated until a fix-point is reached, or a user imposed bound on the number of iterations is reached. In the latter case, the abstraction might be too coarse to prove the given property. However, no automatic refinement procedure is described. The MAGIC 
tool [5] also makes use of weakest pre-conditions in a similar way. Both approaches have the disadvantage that the number of predicates tracked at each program location can be much higher, which may make the single model checking step difficult. In contrast, we propagate the weakest pre-conditions lazily, that is, only to the extent needed to remove infeasible traces. In order to check if a sequence of statements in the $\mathrm{C}$ program is (in)feasible we use a SAT-solver as in [16]. The relationships between a set of predicates is found by making use of SAT-based predicate abstraction [6, 17]. We further improve the performance of SAT-based simulation of counterexamples and abstraction computation by making use of range analysis techniques [19,21] to determine the maximum number of bits needed to represent each variable in the given program.

In the experiments presented in Section 5, F-SoFT computes a single BDD representing the reachable set of states. As is done in SLAM for example, F-SoFT is able to partition the BDD into subsets according to the basic blocks. However, the effects discussed in this paper still carry over to such a scheme as the individual BDDs will be smaller and contain fewer state variables in the support set. This is due to the fact that prior approaches cannot quantify out uninteresting predicates since their value may be important in following basic blocks. The information computed in our approach gives us a more accurate classification of which predicates are useful in a given basic block.

Outline: The following section describes the pre-processing of the source code with our software verification tool F-SoFT [15] and the localized abstraction refinement framework based on weakest pre-condition propagation. F-SoFT allows both SAT-based and BDD-based bounded and unbounded model checking of C. Here, we focus on our BDDbased model checker since BDDs often work well enough for abstract models with few state variables. The third section presents an overview of the computation of the abstraction with and without register sharing, while the fourth section describes our approach of dedicating abstract state variables to predicates. Section 5 discusses the experimental results, and we finish this paper with some concluding remarks.

\section{A Localized Abstraction-Refinement Framework}

\subsection{Software Modeling}

In this section, we briefly describe our software modeling approach that is centered around basic blocks as described in [15]. The preprocessing of the source code is performed before the abstraction refinement routine is invoked. A program counter variable is introduced to monitor progress in the control flow graph consisting of basic blocks. Our modeling framework allows bounded recursion through the introduction of a fixed depth function call stack, when necessary, and introduces special variables representing function return points for non-recursive functions. Due to space limitation, we omit the details of our handling of pointer variables, which can be found in [15]. It is based on adding simplified pointer-free assignments in the basic blocks. 


\subsection{Localization Information}

The formula $\phi$ describes a set of program states, namely, the states in which the value of program variables satisfy $\phi$. The weakest pre-condition [10] of a formula $\phi$ with respect to a statement $s$ is the weakest formula whose truth before the execution of $s$ entails the truth of $\phi$ after $s$ terminates. We denote the weakest pre-condition of $\phi$ with respect to $s$ by $W P(\phi, s)$. Let $s$ be an assignment statement of the form $\mathrm{V}=\mathrm{e}$; and $\phi$ be a $\mathrm{C}$ expression. Then the weakest pre-condition of $\phi$ with respect to $s$, is obtained from $\phi$ by replacing every occurrence of $v$ in $\phi$ with $e$.

Given an if statement with condition $p$, we write assume $\mathrm{p}$ or assume $\neg \mathrm{p}$, depending upon the branch of the if statement that is executed. The weakest pre-condition of $\phi$ with respect to assume $\mathrm{p}$, is given as $\phi \wedge p$. As mentioned earlier, pointer assignments are rewritten early on in our tool chain, thus allowing us to focus here on only the above cases. The weakest pre-condition operator is extended to a sequence of statements by $W P\left(\phi, s_{1} ; s_{2}\right)=W P\left(W P\left(\phi, s_{2}\right), s_{1}\right)$. A sequence of statements $s_{1} ; \ldots ; s_{k}$ is said to be infeasible, if $W P\left(\right.$ true,$\left.s_{1} ; \ldots ; s_{k}\right)=$ false. Note that for ease of presentation, we present the following material using individual statements while the actual implementation uses a control flow graph consisting of basic blocks.

We define $\operatorname{child}(s)$ to denote the set of statements reachable from $s$ in one step in the control flow graph. Each statement $s$ in the program keeps track of the following information: (1) A set of predicates denoted as $\operatorname{local}(s)$ whose values need to be tracked before the execution of $s$. We say a predicate $p$ is active at the statement $s$, if $p \in$ $\operatorname{local}(s)$. (2) A set of predicate pairs denoted as transfer $(s)$. Intuitively, if $\left(p_{i}, p_{j}\right) \in$ transfer $(s)$, then the value of $p_{j}$ after $s$ terminates is equal to the value of $p_{i}$ before the execution of $s$. Formally, a pair $\left(p_{i}, p_{j}\right) \in \operatorname{trans} f \operatorname{er}(s)$ satisfies the following conditions:

- $p_{i} \in\{$ True, False $\} \cup \operatorname{local}(s)$.

- There exists $s^{\prime} \in \operatorname{child}(s)$, such that $p_{j} \in \operatorname{local}\left(s^{\prime}\right)$.

- If $s$ is an assignment statement, then $p_{i}=W P\left(p_{j}, s\right)$.

- If $s$ is an assume statement, then $p_{i}=p_{j}$.

We refer to the sets local $(s)$ and transfer $(s)$ together as the localization information at the statement $s$. This information is generated during the refinement step, and is used for creating refined abstractions which eliminate infeasible traces.

Example: Consider the code in Fig. 1 (a) and the localization information in Fig. 1(d). Since $\left(p_{4}, p_{3}\right) \in \operatorname{transfer}\left(s_{1}\right)$ and $s_{1}$ is an assignment, it means that $p_{4}(c=m)$ is the weakest pre-condition of $p_{3}(x=m)$ with respect to statement $s_{1}$. The value of predicate $p_{4}$ is useful only before the execution of $s_{1}$. After the execution of $s_{1}$, predicate $p_{3}$ becomes useful.

\subsection{Refinement Using Weakest Pre-condition Propagation}

Let $s_{1} ; \ldots ; s_{k}$ be an infeasible program trace. If $s_{i}$ is of the form assume $p_{i}$, then the weakest pre-condition of $p_{i}$ is propagated backwards from $s_{i}$ until $s_{1}$. When computing the weakest pre-condition of a predicate $p_{i}$ with respect to a statement $s_{j}$ of the form assume $p_{j}$, we propagate the weakest pre-conditions of $p_{i}$ and $p_{j}$ separately. That is, 
S

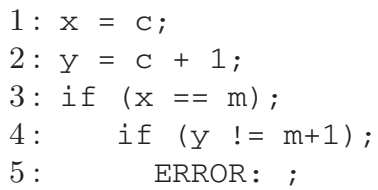

(a)

(c)
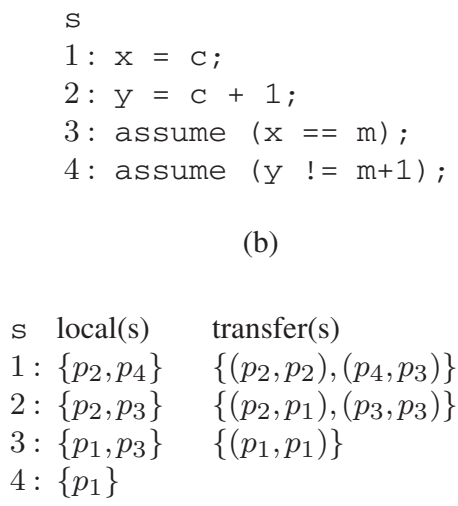

(d)

Fig. 1. (a) A simple C program. (b) An infeasible program trace. (c) Status of local $(s)$ and transfer $(s)$ sets after the first iteration of the refinement algorithm (see Fig. 2). Predicates $p_{1}, p_{2}$ denote $y \neq m+1$ and $c \neq m$, respectively. (d) New additions to the local(s) and transfer $(s)$ in the second iteration. $p_{3}, p_{4}$ denote $x=m$ and $c=m$, respectively

we do not introduce a new predicate for $p_{i} \wedge p_{j}$. This is done to ensure that the predicates remain atomic. The local and the transfer sets for the various statements are updated during this process. The complete algorithm is given in Fig. 2

Example: Consider the C program in Fig. 1(a) and an infeasible trace in Fig. 1(b). Assume that initially local $(s)$ and transfer $(s)$ sets are empty for each $s$. The refinement algorithm in Fig. 2 is applied to the infeasible trace. The localization information after the first iteration $(i=4)$ and second iteration $(i=3)$ of the outer loop in the refinement algorithm, is shown in Fig. 1(c) and Fig. 1), respectively. No change occurs to the localization information for $i=2$ and $i=1$, since $s_{2}$ and $s_{1}$ do not correspond to assume statements.

If $s_{1} ; \ldots ; s_{k}$ is infeasible, then $W P\left(\right.$ true,$\left.s_{1} ; \ldots ; s_{k}\right)=$ false by definition. Intuitively, the atomic predicates in $W P\left(\operatorname{true}, s_{1} ; \ldots ; s_{k}\right)$ appear in $\operatorname{local}\left(s_{1}\right)$. Thus, by finding the relationships between the predicates in $\operatorname{local}\left(s_{1}\right)$, it is possible to construct a refined model which eliminates the infeasible trace. When an infeasible trace $s_{1} ; \ldots ; s_{k}$ is refined using the algorithm in Fig. 2. $s_{1}$ is stored into a set of statements denoted by marked. If a statement $s$ is in the marked set, and the size of $\operatorname{local}(s)$ is less than a certain threshold, then the abstraction routine computes the relationships between the predicates in local $(s)$ using SAT-based predicate abstraction [6, 17]. Otherwise, these relationships are determined lazily by detection of spurious abstract states [1].

Proof Based Analysis: The refinement algorithm described in Fig. 2 2 performs a backward weakest pre-condition propagation for each assume statement in the infeasible trace. However, neither all as sume statements nor all assignments may be necessary for the infeasibility of the given trace. Propagating the weakest pre-conditions for all such statements results in an unnecessary increase in the number of predicates active at each 


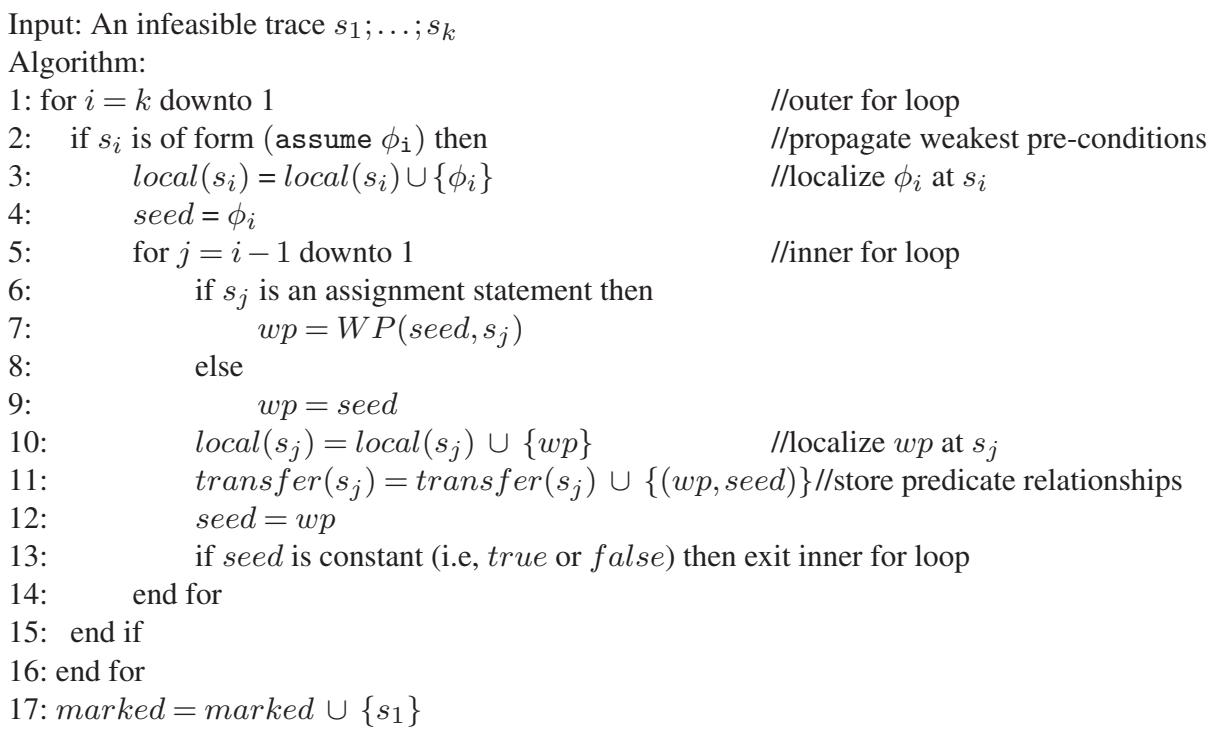

Fig. 2. Predicate localization during refinement

statement in the infeasible trace. We make use of the SAT-based proof of infeasibility of the given trace to determine the statements for which the weakest pre-condition propagation should be done [12]. Thus, the localization information is updated partially, in a way that is sufficient to remove the spurious behavior. The computation of an abstract model using the localization information is described in the next section.

\section{Computing Abstractions}

We describe the abstraction of the given $\mathrm{C}$ program by defining a transition system $T$. The transition system $T=(Q, I, R)$ consists of a set of states $Q$, a set of initial states $I \subseteq Q$, and a transition relation $R\left(q, q^{\prime}\right)$, which relates the current state $q \in Q$ to a next-state $q^{\prime} \in Q$. The abstract model preserves the control flow in the original C program. Let $P=\left\{p_{1}, \ldots, p_{k}\right\}$ denote the union of the predicates active at various program locations. We first describe an abstraction scheme where each predicate $p_{i}$ is assigned one unique Boolean variable $b_{i}$ in the abstract model. The state space of the abstract model is $|L| \cdot 2^{k}$, where $L$ is the set of control locations in the program. We call this scheme abstraction without register sharing. Next, we describe a scheme where the number of Boolean variables needed to represent the predicates in $P$ is equal to the maximum number of predicates active at any program location. The size of the abstract model is given by $|L| \cdot 2^{k^{\prime}}$, where $k^{\prime}=\max _{1 \leq i \leq|L|}\left|\operatorname{local}\left(s_{i}\right)\right|$. We call this scheme abstraction with register sharing. Due to the localization of predicates, $k^{\prime}$ is usually much smaller than $k$, which enables faster model checking of the abstraction obtained using register sharing. 


\subsection{Abstraction Without Register Sharing}

Let $P C$ denote the vector of state variables used to encode the program counter. In abstraction without register sharing each predicate $p_{i}$ has a state variable $b_{i}$ in the abstract model. Each state in the abstraction corresponds to the valuation of $|P C|+k$ state variables, where $k$ is the total number of predicates. In the initial state $P C$ is equal to the value of the entry location in the original program. The state variables corresponding to the predicates are initially assigned non-deterministic Boolean values. Given a statement $s_{l}$ and a predicate $p_{i}$ the following cases are possible:

- $s_{l}$ is either an assume statement or an assignment statement that does not assign to any variable in $p_{i}$. That is, after executing $s_{l}$ the value of predicate $p_{i}$ remains unchanged. Thus, in the abstract model the value of the state variable $b_{i}$ remains unchanged after executing $s_{l}$. We denote the set of all statements where $p_{i}$ is unchanged as unc $\left(p_{i}\right)$.

- $s_{l}$ assigns to some variable in $p_{i}$. Let $p_{j}$ denote the weakest pre-condition of $p_{i}$ with respect to $s_{l}$. If the predicate $p_{j}$ is active at $s_{l}$, that is $p_{j} \in \operatorname{local}\left(s_{l}\right)$, and $\left(p_{j}, p_{i}\right) \in$ transfer $\left(s_{l}\right)$, then after executing $s_{l}$, the value of predicate $p_{i}$ is the same as the value of predicate $p_{j}$ before executing $s_{l}$. In the abstract model this simply corresponds to transferring the value of $b_{j}$ to $b_{i}$ at $s_{l}$. If the predicate $p_{j}$ is not active at $s_{l}$, then the abstract model assigns a non-deterministic Boolean value to $b_{i}$ at $s_{l}$. This is necessary to ensure that the abstract model is an over-approximation of the original program.

We denote the set of all statements that can update the value of a predicate $p_{i}$ as update $\left(p_{i}\right)$. The set of statements where the weakest pre-condition of $p_{i}$ is available is denoted by $w p a\left(p_{i}\right)$. Using the localization information from Sec. 2.2. $w p a\left(p_{i}\right)$ is defined as follows: $w p a\left(p_{i}\right):=\left\{s_{l} \mid s_{l} \in \operatorname{update}\left(p_{i}\right) \wedge \exists p_{j} .\left(p_{j}, p_{i}\right) \in\right.$ transfer $\left.\left(s_{l}\right)\right\}$.

We use $\operatorname{inp}\left(p_{i}\right)$ to denote the set of statements that assign a non-deterministic value $v_{i}$ to the state variable $b_{i}$. This set is defined as update $\left(p_{i}\right) \backslash w p a\left(p_{i}\right)$. Let $c_{i l}$ denote the state variable corresponding to the weakest pre-condition of predicate $p_{i}$ with respect to $s_{l}$. We use $p c_{l}$ to denote that the program counter is at $s_{l}$, that is $P C=l$, and $v_{i}$ to denote a non-deterministic input variable. The next state function for the variable $b_{i}$ is then defined as follows:

$$
b_{i}^{\prime}:=\left[\bigvee_{\mathrm{s}_{l} \in \operatorname{unc}\left(p_{i}\right)}\left(p c_{l} \wedge b_{i}\right)\right] \vee\left[\bigvee_{\left.\mathrm{s}_{l} \in \text { wpa(p } p_{i}\right)}\left(p c_{l} \wedge c_{i l}\right)\right] \vee\left[\bigvee_{\mathrm{s}_{l} \in \operatorname{inp}\left(p_{i}\right)}\left(p c_{l} \wedge v_{i}\right)\right]
$$

Note that no calls to a decision procedure are needed when generating the next-state functions. All the required information is gathered during the refinement step itself by means of weakest pre-condition propagation.

Example: Consider the abstraction of the program in Fig. [3 a) with respect to the localization information given in Fig. 3 b b). The predicate $p_{1}(y \neq m+1)$ is updated at statement $s_{2}$, and its weakest pre-condition $p_{2}(c \neq m)$ is active at $s_{2}$, and $\left(p_{2}, p_{1}\right) \in$ transfer $\left(s_{2}\right)$. So the next state function for the state variable representing $p_{1}$ is given as follows: $b_{1}^{\prime}:=\left(p c_{2} \wedge b_{2}\right) \vee\left(\left(p c_{1} \vee p c_{3} \vee p c_{4}\right) \wedge b_{1}\right)$. The other next state functions are given as follows: $b_{2}^{\prime}:=b_{2}, b_{4}^{\prime}:=b_{4}$, and $b_{3}^{\prime}:=\left(p c_{1} \wedge b_{4}\right) \vee\left(\left(p c_{2} \vee p c_{3} \vee p c_{4}\right) \wedge b_{3}\right)$. The resulting abstraction is shown in Fig. 3](c). For simplicity the control flow is shown explicitly in the abstraction. 


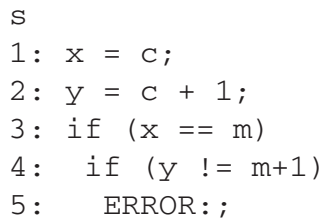

(a)

\author{
local(s) transfer(s) \\ $\left\{p_{2}, p_{4}\right\} \quad\left\{\left(p_{2}, p_{2}\right),\left(p_{4}, p_{3}\right)\right\}$ \\ $\left\{p_{2}, p_{3}\right\} \quad\left\{\left(p_{2}, p_{1}\right),\left(p_{3}, p_{3}\right)\right\}$ \\ $\left\{p_{1}, p_{3}\right\} \quad\left\{\left(p_{1}, p_{1}\right)\right\}$ \\ $\left\{p_{1}\right\}$
}

Abstraction

1: skip;

2: skip;

3: if $\left(b_{2}\right)$

4: if $\left(b_{1}\right)$

5: ERROR: ; (e)
Abstraction

1: $b_{3}=b_{4}$;

2: $b_{1}=b_{2}$;

3: if $\left(b_{3}\right)$

4: if $\left(b_{1}\right)$

5: ERROR: ;

(c)
s Mapping
1: $\left\{p_{2}: b_{1}, p_{4}: b_{2}\right\}$
2: $\left\{p_{2}: b_{1}, p_{3}: b_{2}\right\}$
3: $\left\{p_{1}: b_{1}, p_{3}: b_{2}\right\}$
4: $\left\{p_{1}: b_{1}\right\}$
5:

(d)

Fig. 3. (a) C program. (b) Localization information for the program where $p_{1}, p_{2}, p_{3}, p_{4}$ denote the predicates $y \neq m+1, c \neq m, x=m, c=m$, respectively. (c) Abstraction with no register sharing. Boolean variable $b_{i}$ represents the value of $p_{i}$ in the abstraction. (d) Mapping of predicates in $\operatorname{local}(s)$ for each $s$ to the Boolean variables (register sharing). (e) Abstraction with register sharing. (f) Global constraint and Local constraint for abstractions in (c) and (e) , respectively

Global Constraint Generation: The precision of the abstraction can be increased by finding the relationships between the predicates in $\operatorname{local}(s)$ for some $s$. For example, in Fig. 3 (b) the relationship between the predicates in $\operatorname{local}\left(s_{1}\right)$ results in a global constraint, $b_{2} \leftrightarrow \neg b_{4}$. This constraint holds in all states of the abstract model of Fig. 3 (c) as the Boolean variables $b_{2}$ and $b_{4}$ always represent the same predicate throughout the abstraction without register sharing. The abstraction without register sharing given in Fig. 3 (c) combined with the global constraint in Fig. 3 (f) is sufficient to show that the ERROR label is not reachable in the $\mathrm{C}$ program given in Fig. 3 (a). Note that we could have simplified the computation here by recognizing that $p_{4}=\neg p_{2}$, which we omit for presentation purposes only.

The constraint generation is done only for some of the statements which are marked during the refinement (Fig. 2 line no. 17). We use SAT-based predicate abstraction [6, 17] to find the relationships between the predicates in $\operatorname{local}(s)$ for such statements. This is the only time we use any decision procedure other than checking for the feasibility of traces. Due to the computational cost of enumerating the set of solutions, we only perform this computation for very small sets of predicates. Other relationships are then discovered on demand based on spurious abstract states [1].

\subsection{Abstraction with Register Sharing}

In abstraction with no register sharing, the state-space of the abstract model is $|L| \cdot 2^{|P|}$, where $P$ is the set of predicates, and $L$ is the set of locations in the given program. Thus, when the number of predicates is large, model checking of the abstraction can become a bottleneck even with a symbolic representation of the state space. We make use of the 
locality of predicates to speed up the model checking of the abstraction. This is done by reducing the number of (Boolean) state variables in the abstraction. The fact that each state variable in the abstract model is only locally useful can be used to represent different predicates in different parts of the program using the same state variable. We call the reuse of state variables in the abstract model register sharing.

Example: Consider the $\mathrm{C}$ program in Fig. 3 (a) and the localization information in Fig. 3b). The abstraction of this program with no register sharing in Fig. 3] c), contains four state variables, one for each predicate. However, the number of predicates active at any program statement is $\max _{1 \leq i \leq 4}\left|\operatorname{local}\left(s_{i}\right)\right|=2$. Intuitively, it should be possible to create an abstraction with just two state variables.

The predicates $p_{2}, p_{4}$ are active at program location 1 , so we introduce two Boolean variables $b_{1}, b_{2}$, to represent each of these predicates, respectively. After the execution of $s_{1}$, predicate $p_{4}$ is no longer active, and the state variable $b_{2}$ can be used to represent some other predicate. Predicate $p_{3}$ becomes active at $s_{2}$, so we can reuse the abstract variable $b_{2}$ to represent $p_{3}$ at $s_{2}$. In a similar fashion, $b_{1}$ can be reused to represent predicate $p_{1}$ at program locations $s_{3}$ and $s_{4}$. We use $p: b$ to denote that the predicate $p$ is represented by the state variable $b$. The mapping of active predicates at each program location to the state variables is given in Fig 3 (d).

The abstraction with register sharing is obtained by translating the predicate relationships in transfer $(s)$ for each $s$, according to the mapping discussed above. Continuing our example, $\left(p_{4}, p_{3}\right) \in$ transfer $\left(s_{1}\right)$ in Fig. 3 $\left.\mathrm{b}\right)$, the value of the state variable representing $p_{4}$ at $s_{1}$, must be transferred to the state variable representing $p_{3}$, afterwards. Since both $p_{4}$ and $p_{3}$ are represented by the same state variable $b_{2}$, the abstraction for $s_{1}$ does not alter the value of $b_{2}$. The abstraction using only two state variables $\left(b_{1}, b_{2}\right)$ is shown in Fig 3 (e). The skip statement means that the values of the state variables $b_{1}$ and $b_{2}$ remain unchanged for that statement.

Mapping Predicates to State Variables: Recall, that $p=\left\{p_{1}, \ldots, p_{k}\right\}$ denotes the set of predicates. Let $B=\left\{b_{1}, \ldots, b_{l}\right\}$ be the set of state variables in the abstraction, where $l$ equals the maximum number of active predicates at any program location. For every statement $s$, the predicates relevant at $s$ are mapped to unique state variables in $B$. Let map be a function that takes a statement $s$ and a predicate $p$ as arguments. If $p \in \operatorname{local}(s)$, then the result of $\operatorname{map}(s, p)$ is a state variable $b \in B$; otherwise, the result is $\perp$. Recall that $\operatorname{child}(s)$ denotes the set of statements reachable from $s$ in one step in the control flow graph. The constraints to be satisfied by map are as follows:

- Two distinct predicates which are active together at the same statement should not be assigned the same Boolean variable in the abstraction for that statement.

$$
\forall s \forall p_{i}, p_{j} \in \operatorname{local}(s)\left[p_{i} \neq p_{j} \rightarrow \operatorname{map}\left(s, p_{i}\right) \neq \operatorname{map}\left(s, p_{j}\right)\right]
$$

- Consider statement $s$ and $\left(p_{1}, p_{2}\right) \in \operatorname{transfer}(s)$. By definition there exists $s^{\prime} \in$ $\operatorname{child}(s)$ where $p_{2}$ is active, that is $p_{2} \in \operatorname{local}\left(s^{\prime}\right)$. This case is shown in Fig. 4 (a). Suppose the predicate $p_{1}$ is mapped to $b_{i}$ in $s$ and $p_{2}$ is mapped to $b_{j}$ in $s^{\prime}$. The abstraction for the statement $s$ will assign the value of $b_{i}$ to $b_{j}$. So $b_{j}$ should not be 


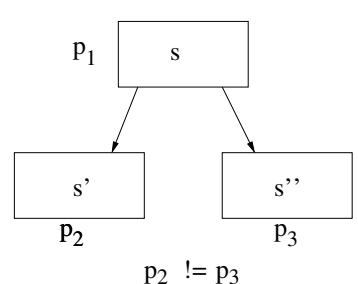

(a)

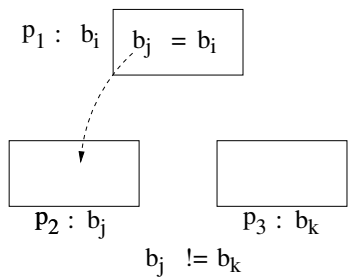

(b)

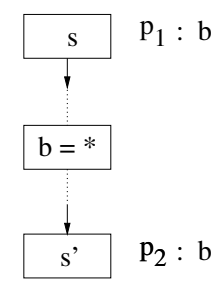

(c)

Fig. 4. (a) Statement $s$ and two successors $s^{\prime}$ and $s^{\prime \prime}$. Predicates $p_{1}, p_{2}, p_{3}$ are active at $s, s^{\prime}$, and $s^{\prime \prime}$, respectively. (b) Abstraction with register sharing, where $\left(p_{1}, p_{2}\right) \in \operatorname{transfer}(s)$. Predicate $p_{1}, p_{2}$ are mapped to $b_{i}, b_{j}$, respectively, in the abstraction. Predicate $p_{3} \neq p_{2}$ should not be mapped to $b_{j}$ for safe abstraction i.e., an over-approximation of the original program. (c) Boolean variable $b$ is used to represent two distinct predicates $p_{1}$ and $p_{2}$ on the same path. It is set to a * (non-deterministic value) between $s$ and $s^{\prime}$ to ensure safe abstraction

used to represent a predicate $p_{3}$, where $p_{3} \neq p_{2}$, in any other successor of $s$. This is because there is no relationship between the value of the predicate $p_{1}$ at $s$ and the predicate $p_{3}$ at $s^{\prime \prime}$. This constraint is shown in Fig. 4(b).

We now describe the algorithm which creates an abstraction in the presence of register sharing. Let $a b s(s)$ be a set of Boolean pairs associated with each statement $s$. Intuitively, if $\left(b_{l}, b_{m}\right) \in a b s(s)$, then in the abstraction the value of $b_{m}$ after $s$ terminates is equal to the value of $b_{l}$ before the execution of $s$. Formally, $a b s(s)$ is defined as follows:

$$
\begin{gathered}
\operatorname{abs}(s):=\left\{\left(b_{l}, b_{m}\right) \mid \exists\left(p_{i}, p_{j}\right) \in \text { transfer }(s) . b_{l}=\operatorname{map}\left(s, p_{i}\right) \wedge\right. \\
\left.\exists s^{\prime} \in \operatorname{child}(s) . b_{m}=\operatorname{map}\left(s^{\prime}, p_{j}\right)\right\} .
\end{gathered}
$$

Given a Boolean variable $b_{i}$ and a statement $s_{l}$, the following cases are possible:

- $s_{l}$ updates the value of $b_{i}$. That is, there exists a $b_{j} \in B$ such that $\left(b_{j}, b_{i}\right) \in a b s\left(s_{l}\right)$. We denote the set of all statements which update $b_{i}$ as update $\left(b_{i}\right)$. The function $r h s\left(s_{l}, b_{i}\right)$ returns the Boolean variable which is assigned to $b_{i}$ in the statement $s_{l}$.

- $s_{l}$ assigns a non-deterministic value to $b_{i}$. The set of all such statements is denoted by nondet $\left(b_{i}\right)$. In order to understand the use of this set, consider a Boolean variable $b$ which is used to represent two distinct predicates $p_{1}$ and $p_{2}$ on the same path. Assume that $b$ is not used to represent any other predicate between the statements $s$ and $s^{\prime}$. Since $p_{1}$ and $p_{2}$ are not related, the value of $b$ when it is representing $p_{1}$ should not be used when $b$ is representing $p_{2}$. So $b$ is assigned a non-deterministic value between the path starting from $s$ to $s^{\prime}$. This is necessary to ensure that the abstraction is an over-approximation of the original program. This case is shown in Fig. 4 (c).

- The value of $b_{i}$ is a don't-care at statement $s_{l}$. The value of $b_{i}$ is a don't care for all the statements which are not present in update $\left(b_{i}\right)$ or nondet $\left(b_{i}\right)$. In such cases, we set the value of $b_{i}$ to false at these statements, in order to simplify its conjunction with the program counter variable to false. This simplifies the overall transition relation. 
Given the above information the next state function for the variable $b_{i}$ is defined as follows (we use an input $v_{i}$ for introducing non-determinism and $p c_{l}$ to denote $P C=l$ ):

$$
b_{i}^{\prime}:=\left[\bigvee_{\mathrm{s}_{l} \in \operatorname{update}\left(b_{i}\right)}\left(p c_{l} \wedge r h s\left(s_{l}, b_{i}\right)\right)\right] \vee\left[\bigvee_{\mathrm{s}_{l} \in \operatorname{nondet}\left(p_{i}\right)}\left(p c_{l} \wedge v_{i}\right)\right]
$$

Local constraint generation: The abstraction can be made more precise by relating the predicates in local $(s)$ for some $s$. For example, in Fig. 3 (b) the predicates in local $\left(s_{1}\right)$ satisfy the constraint that $p_{2} \leftrightarrow \neg p_{4}$. In order to add this constraint to the abstraction, we need to translate it in terms of the Boolean variables. The mapping given in Fig.3 (d) assigns Boolean variables $b_{1}, b_{2}$ to $p_{2}, p_{4}$, at $s_{1}$ respectively. This leads to a constraint $(P C=1) \rightarrow\left(b_{1} \leftrightarrow \neg b_{2}\right)$. This is called a local constraint as it is useful only when $P C=1$. We cannot omit the $P C=1$ term from the constraint as this would mean that $b_{1} \leftrightarrow \neg b_{2}$ holds throughout the abstraction. The abstraction with register sharing in Fig. 3(e) combined with the local constraint in Fig. 3 (f) is sufficient to show that the ERROR label is not reachable in the C program given in Fig. 3. a).

\section{Dedicated State Variables}

Register sharing enables the creation of abstract models with as few Boolean variables as possible which enables more efficient model checking of the abstractions. However, register sharing might also result in a large number of refinement iterations as described in the following. Consider a sequence $S E$ of statements from $s$ to $s^{\prime}$, which does not modify the value of a predicate $p$. Suppose $p$ is localized at the statements $s, s^{\prime}$, but not at any intermediate statement in $S E$. In abstraction with register sharing, it is possible that $p$ is represented by two different Boolean variables $b_{1}$ and $b_{2}$ at $s$ and $s^{\prime}$, respectively. Because the value of $p$ remains unchanged along $S E$, the value of $b_{1}$ at $s$ should be equal to the value of $b_{2}$ at $s^{\prime}$. If this is not tracked, we may obtain a spurious counterexample by assigning different values to $b_{1}$ at $s$ and $b_{2}$ at $s^{\prime}$. This leads to a refinement step, which localizes the predicate $p$ at every statement in $S E$, to ensure that the value of predicate $p$ does not change along $S E$ in subsequent iterations. We should note that such behavior is handled in the abstraction without register sharing approach through the use of the unchanged set denoted by unc in Eqn. (1) described earlier.

If $p$ is discovered frequently in different parts of the program through various spurious counterexamples, then using the abstraction with register sharing will lead to many abstraction refinement iterations. This problem can be avoided, if $p$ is represented by exactly one Boolean variable $b$ in a large scope of the abstraction. This is because the value of $b$ will not be changed by any statement in $S E$, and thus, the value of $b$ at $s^{\prime}$ will be the same as that at $s$. We call a Boolean variable which represents only one predicate for a large scope a dedicated state variable. The next state function for a dedicated state variable $b$ is computed using Eqn. (1).

Hybrid Approach: Initially, when a predicate is discovered it is assigned a Boolean variable, which can be reused for representing different predicates in other parts of the abstraction. If the same predicate is discovered through multiple counterexamples in the 
various parts of the program, then it is assigned a dedicated Boolean variable for a global or functional scope of the program depending on the variables used in the predicate. The decision about when to assign a dedicated Boolean variable to a predicate is done by making use of the following heuristic.

For each predicate $p$, let $\operatorname{usage}(p, i)$ denote the number of statements where $p$ is localized in the iteration number $i$ of the abstraction refinement loop. If usage $(p, i)$ exceeds a certain user-defined threshold $T H$, then $p$ is assigned a dedicated Boolean variable. If $T H=0$, then every predicate will be assigned a dedicated state variable as soon as it is discovered. This is similar to performing abstraction with no register sharing for all state variables. On the other hand, if $T H=|L|+1$, where $|L|$ is the total number of statements in the program, then none of the predicates will be assigned a dedicated state variable. This allows complete reuse of the abstract variables, which is similar to abstraction with register sharing. For any intermediate value of $T H$ we have a hybrid of abstraction with and without register sharing.

In the hybrid approach, it is possible to have global constraints on the dedicated state variables. This saves refinement iterations where the same constraint is added locally in various parts by means of counterexamples. We can still have local constraints on the state variables which are reused. Furthermore, we hope to discover as early as possible whether a predicate should be given a dedicated state variable by having a low threshold for the early iterations of the abstraction refinement loop, which increases as the number of iterations increases. Predicting early on that a predicate may need a dedicated state variable reduces the number of abstraction refinement iterations substantially.

\section{Experimental Results}

We have implemented these techniques in NEC's F-SoFT [15] verification tool. All experiments were performed on a $2.8 \mathrm{GHz}$ dual-processor Linux machine with $4 \mathrm{~GB}$ of memory. We report our experimental results on the TCAS and Alias case studies. TCAS (Traffic Alert and Collision Avoidance System) is an aircraft conflict detection and resolution system used by all US commercial aircrafts. We used an ANSI-C version of a TCAS component available from Georgia Tech. Even though the pre-processed program has only 1652 lines of code, the number of predicates needed to verify the properties is non-trivial for both F-SoFT and BLAST. We checked 10 different safety properties of the TCAS system. Alias is an artificial benchmark which makes extensive use of pointers. Each property was encoded as a certain error label in the code. If the label is not reachable, then the property is said to hold. Otherwise, we report the length of the counterexample in the "Bug" column in Table 1 CPU times are given in seconds, and we set a time limit of one hour for each analysis. Note, that many implementation details of F-SofT and BLAST not discussed here may impact the measured runtimes.

\subsection{Predicate Localization, Register Sharing, and Dedicated State Variables}

We first experimented with no localization of predicates. However, this approach did not scale, as the abstraction computation becomes a bottleneck. We next experimented with localization of predicates using weakest pre-conditions. The results of applying 
only localization and abstraction without register sharing is shown under the "Localize" heading in the Table 1 The "Time Abs MC" column gives the total time, followed by the breakup of total time into the time taken by abstraction (Abs), model checking (MC), respectively. We omit the time taken by refinement, which is equal to Time - (Abs + $\mathrm{MC}$ ) for each row. The "P" and the "I" columns give the total number of predicates, and the total number of iterations, respectively. Two observations can be made from the "Localize" results: 1) Due to the localization of predicates, the abstraction computation is no longer a bottleneck. 2) Model checking takes most of the time, since for each predicate a state variable is created in the abstract model. Note that the model checking step is the cause of the timeouts in three rows under the "Localize" results.

Next, we experimented with register sharing. The number of state variables in the abstraction was reduced, and the individual model-checking steps became faster. However, as discussed in Sec. 4 this approach resulted in too many abstraction refinement iterations. This problem was solved by discovering on-the-fly whether a predicate should be assigned a dedicated state variable, that is, a state variable which will not be reused. A dedicated state variable is introduced for a predicate whose usage exceeds a progressively increasing threshold, starting at $5 \%$ of the total number of program locations.

The results of combining these multiple techniques is given under the "Combined" heading in Table 1. The "P Max Ded" column gives the total number of predicates (P), followed by the maximum number of predicates active at any program location (Max), and the total number of state variables which represent exactly one predicate, that is, dedicated state variables (Ded). Observe that the time spent during model checking (MC) has reduced significantly as compared to the "Localize" column.

We also experimented with the $T H$ (threshold) parameter, which is used to determine when a predicate is assigned a dedicated state variable. Fig. 5(a) shows the variation of the total runtime with the initial value for the threshold. When the threshold is equal to zero every predicate is assigned a dedicated state variable. This results in too many state variables in the abstract model causing the total runtime to be high. However, as the

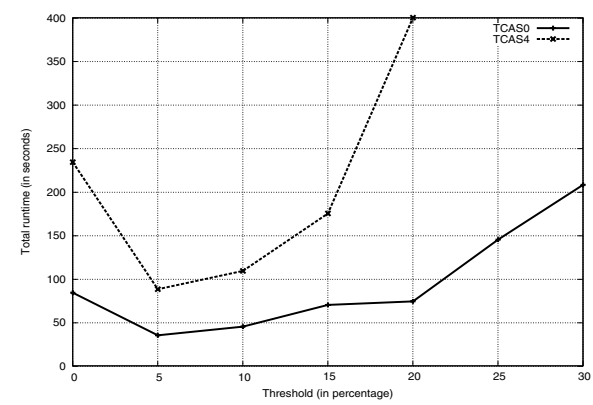

(a)

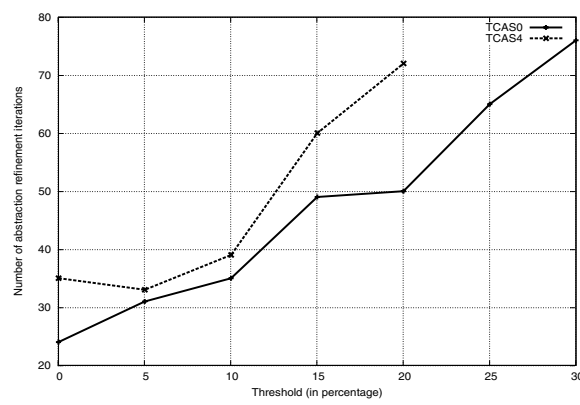

(b)

Fig. 5. (a) Variation in the total runtime with the threshold. (b) Variation in the total number of abstraction refinement iterations with the threshold 
Table 1. Results for: 1) Localization, abstraction without register sharing ("Localize") . 2) Localization, abstraction with register sharing, dedicated state variables ("Combined"). 3) BLAST with interpolation ("BLAST"). A "-" indicates that the property holds. A "." indicates that the benchmark could not be handled properly. A "TO" indicates a timeout of $1 \mathrm{hr}$. We report the statistics observed before timeout occurs

\begin{tabular}{|c|c|c|c|c|c|c|c|c|c|c|c|c|c|c|c|c|c|c|}
\hline \multirow{2}{*}{$\begin{array}{l}\text { Bench } \\
\text {-mark }\end{array}$} & \multicolumn{5}{|c|}{ Localize } & \multicolumn{7}{|c|}{ Combined } & \multicolumn{5}{|c|}{ BLAST } & \multirow[t]{2}{*}{ Bug } \\
\hline & Time & Abs & $\mathrm{MC}$ & $\mathrm{P}$ & I & Time & $\mathrm{Abs}$ & $\mathrm{MC}$ & $\mathrm{P}$ & Max & Ded & I & Time & $\mathrm{P}$ & Max & Avg & I & \\
\hline TCASO & 245 & 7 & 196 & 71 & 32 & 36 & 5 & 15 & 65 & 26 & 18 & 31 & 96 & 85 & 24 & 10 & 33 & - \\
\hline TCAS1 & 1187 & 15 & 1069 & 108 & 44 & 161 & 9 & 118 & 96 & 35 & 25 & 38 & 256 & 137 & 43 & 17 & 42 & - \\
\hline TCAS2 & 952 & 10 & 882 & 74 & 38 & 104 & 25 & 51 & 95 & 31 & 24 & 36 & 148 & 108 & 31 & 11 & 40 & - \\
\hline TCAS3 & 940 & 15 & 864 & 91 & 36 & 46 & 17 & 17 & 73 & 22 & 15 & 33 & 172 & 101 & 26 & 10 & 44 & 152 \\
\hline TCAS4 & 1231 & 13 & 1111 & 97 & 39 & 88 & 9 & 48 & 90 & 34 & 25 & 32 & 182 & 149 & 38 & 13 & 51 & 166 \\
\hline TCAS5 & 1222 & 11 & 1128 & 79 & 41 & 141 & 8 & 98 & 98 & 37 & 29 & 31 & 105 & 114 & 31 & 10 & 33 & - \\
\hline TCAS6 & TO & 20 & 2270 & 117 & 49 & 330 & 16 & 266 & 109 & 40 & 33 & 40 & 293 & 158 & 41 & 14 & 69 & 179 \\
\hline TCAS7 & 1758 & 16 & 1627 & 79 & 47 & 64 & 10 & 29 & 94 & 28 & 21 & 33 & 287 & 125 & 30 & 11 & 63 & 160 \\
\hline TCAS8 & TO & 21 & 1988 & 84 & 51 & 119 & 13 & 68 & 106 & 34 & 27 & 41 & 181 & 116 & 31 & 11 & 46 & - \\
\hline TCAS9 & TO & 26 & 3349 & 113 & 58 & 250 & 14 & 186 & 106 & 34 & 27 & 44 & 322 & 140 & 40 & 14 & 61 & 179 \\
\hline ALIAS & 50 & 6 & 33 & 61 & 11 & 6 & 2 & 1 & 55 & 25 & 15 & 9 & . & . & . & & . & - \\
\hline
\end{tabular}

threshold is increased, the number of abstraction refinement iterations starts to increase as shown in Fig. 5 b). The best runtime in our experiments has so far been obtained for an initial threshold of $5 \%$. Even such a small value for the threshold is effective in separating the predicates which are globally relevant from those which are locally useful. As the threshold is further increased very few predicates are assigned dedicated state variables. One of the main advantages of choosing a small initial threshold is that we are able to decide early on whether a predicate may need a dedicated state variable. If we start with a higher initial threshold, the number of additional iterations needed for a single predicate to receive a dedicated state variable increases too much.

The map function (see Section 3.2) is computed incrementally, as new predicates are discovered. Suppose during refinement a predicate $p$ gets added to local $(s)$ for some $s$. In order to find a state variable to represent the value of $p$ at $s$, we first check if some existing state variable can be reused without violating the constraints described in Section 3.2. Let the total number of times reuse is possible be $R$. If no existing state variable can be used, we introduce a new state variable for representing the value of $p$ at $s$. Let the total number of times a new state variable is introduced be $C$. The ratio $R /(C+R)$ measures the effectiveness of variable reuse in controlling the total number of state variables. The value of this ratio is $88 \%$ on average across the TCAS benchmarks and $81 \%$ for the ALIAS benchmark.

\subsection{Comparison with BLAST}

We first ran BLAST in the default mode without any options. However, the default predicate discovery scheme in BLAST fails to find the new set of predicates during refinement, and terminates without (dis)proving any of the TCAS properties. Next, we tried the Craig interpolation [14] options (craig1 and craig2) provided by BLAST. The BLAST manual recommends the use of predH7 heuristic with Craig interpolation. 
Of the various options to BLAST, craig2 and predH7 result in the best performance when checking the TCAS properties. Table 1 gives the result of running BLAST with these options under the "BLAST" heading. The "P Max Avg" column gives the total number of predicates (P), followed by the maximum (Max) and the average (Avg) number of predicates active at any program location (rounded to the nearest integer).

The best runtimes are shown in bold in Table 1 Note that the "Combined" technique of F-SoFT outperforms BLAST on 9 out of 11 benchmarks, and the number of iterations required by "Combined" is less than that for "BLAST" in all cases. Recall that the size of the abstraction is exponential in the maximum number of active predicates (Max). This number is comparable for both BLAST and F-SoFT, even though BLAST makes use of a more complex refinement technique based on the computation of interpolants.

\section{Conclusions and Future Work}

The application of the predicate abstraction paradigm to large software depends crucially on the choice and usage of the predicates. If all predicates are tracked globally in the program, the analysis often becomes intractable due to the large number of predicate relationships. In this paper we described various techniques for improving the overall performance of the abstraction refinement loop. We presented experimental results in our F-SoFT [15] toolkit using the techniques of predicate localization, register sharing and dedicated state variables, and showed how a combination of these techniques allowed us to check properties requiring a large number of predicates.

There are a number of interesting avenues for future research. Theoretical comparison between the use of interpolants [14] and the use of weakest pre-conditions for localization of predicates will be useful. Other techniques for finding the right balance between the predicates whose values are tracked locally and the predicates whose values are tracked globally are worth further investigation. Furthermore, we need to experiment with these heuristics for more and larger case studies as well.

Acknowledgment. We thank Rupak Majumdar and Ranjit Jhala for their help with BLAST.

\section{References}

1. T. Ball, B. Cook, S. Das, and S. Rajamani. Refining approximations in software predicate abstraction. In TACAS 04, pages 388-403. Springer, 2004.

2. T. Ball, R. Majumdar, T.D. Millstein, and S.K. Rajamani. Automatic predicate abstraction of C programs. In Programming Language Design and Implementation, pages 203-213, 2001.

3. T. Ball, A. Podelski, and S.K. Rajamani. Boolean and Cartesian abstraction for model checking C programs. In TACAS 01, volume 2031, 2001.

4. T. Ball and S.K. Rajamani. Automatically validating temporal safety properties of interfaces. In SPIN Workshop on Model Checking of Software. Springer, 2001.

5. S. Chaki, E. Clarke, A. Groce, S. Jha, and H. Veith. Modular verification of software components in C. In ICSE 03, pages 385-395. IEEE, 2003.

6. E. Clarke, D. Kroening, N. Sharygina, and K. Yorav. Predicate abstraction of ANSI-C programs using SAT. Formal Methods in System Design, 25:105-127, Sep-Nov 2004. 
7. P. Cousot and R. Cousot. Abstract interpretation: a unified lattice model for static analysis of programs by construction or approximation of fixpoints. In Proceedings of the 4th ACM Symposium on Principles of Programming Languages, pages 238-252, 1977.

8. William Craig. Linear reasoning. In Journal of Symbolic Logic, pages 22:250-268, 1957.

9. S. Das, D. Dill, and S. Park. Experience with predicate abstraction. In Computer Aided Verification, LNCS 1633, pages 160-171. Springer, 1999.

10. E. Dijkstra. A Discipline of Programming. Prentice Hall, 1976.

11. S. Graf and H. Saidi. Construction of abstract state graphs with PVS. In $C A V$ 97, pages 72-83. Springer, 1997.

12. A. Gupta, M.K. Ganai, P. Ashar, and Z. Yang. Iterative abstraction using SAT-based BMC with proof analysis. In International Conference on Computer Aided Design (ICCAD), 2003.

13. T. A. Henzinger, R. Jhala, R. Majumdar, and G. Sutre. Lazy abstraction. In POPL O2, pages 58-70, 2002.

14. T.A. Henzinger, R. Jhala, R. Majumdar, and K.L. McMillan. Abstractions from proofs. In POPL 04, pages 232-244. ACM Press, 2004.

15. F. Ivančić, Z. Yang, M. Ganai, A. Gupta, and P. Ashar. Efficient SAT-based bounded model checking for software verification. In Symposium on Leveraging Applications of Formal Methods, 2004.

16. H. Jain, D. Kroening, and E. Clarke. Verification of SpecC using predicate abstraction. In MEMOCODE 04, pages 7-16. IEEE, 2004.

17. S. K. Lahiri, R. E. Bryant, and B. Cook. A symbolic approach to predicate abstraction. In CAV 03, pages 141-153. Springer, 2003.

18. Kedar S. Namjoshi and Robert P. Kurshan. Syntactic program transformations for automatic abstraction. In CAV 00, number 1855 in LNCS, 2000.

19. R. Rugina and M.C. Rinard. Symbolic bounds analysis of pointers, array indices, and accessed memory regions. In PLDI 00, pages 182-195, 2000.

20. Vlad Rusu and Eli Singerman. On proving safety properties by integrating static analysis, theorem proving and abstraction. In TACAS 99, pages 178-192, 1999.

21. A. Zaks, F. Ivančić, H. Cadambi, I. Shlyakhter, Z. Yang, M. Ganai A. Gupta, and P. Ashar. Range analysis for software verification. Submitted for publication, 2005. 